\title{
F-Expansion Method and Its Application for Finding New Exact Solutions to the Kudryashov-Sinelshchikov Equation
}

\author{
Yun-Mei Zhao \\ Department of Mathematics, Honghe University, Mengzi, Yunnan 661199, China \\ Correspondence should be addressed to Yun-Mei Zhao; zhaoyunmei2000@126.com
}

Received 21 January 2013; Accepted 7 April 2013

Academic Editor: Anjan Biswas

Copyright (C) 2013 Yun-Mei Zhao. This is an open access article distributed under the Creative Commons Attribution License, which permits unrestricted use, distribution, and reproduction in any medium, provided the original work is properly cited.

Based on the F-expansion method, and the extended version of $F$-expansion method, we investigate the exact solutions of the Kudryashov-Sinelshchikov equation. With the aid of Maple, more exact solutions expressed by Jacobi elliptic function are obtained. When the modulus $m$ of Jacobi elliptic function is driven to the limits 1 and 0 , some exact solutions expressed by hyperbolic function solutions and trigonometric functions can also be obtained.

\section{Introduction.}

In the recent years, the study of nonlinear partial differential equations (NLEEs) modelling physical phenomena has become an important toll. Seeking exact solutions of NLEEs has long been one of the central themes of perpetual interest in mathematics and physics. With the development of symbolic computation packages like Maple and Mathematica, many powerful methods for finding exact solutions have been proposed, such as homogeneous balance method $[1,2]$, auxiliary equation method $[3,4]$, the Expfunction method [5, 6], Darboux transformation [7, 8], tanhfunction method [9], the modified extended tanh-function [10], and Jacobi elliptic function expansion method [11, $12]$.

The F-expansion method is an effective and direct algebraic method for finding the exact solutions of nonlinear evolution problems [13-15], many nonlinear equations have been successfully solved. Later, the further developed methods named the generalized $F$-expansion method [16, 17], the modified F-expansion method [18], the extended $F$-expansion method [19], and the improved $F$-expansion method [20] have been proposed and applied to many nonlinear problems.

Recently, Kudryashov and Sinelshchikov [21] introduced the following equation:

$$
\begin{aligned}
& u_{t}+\gamma u u_{x}+u_{x x x}-\varepsilon\left(u u_{x x}\right)_{x}-\kappa u_{x} u_{x x}-v u_{x x}-\delta\left(u u_{x}\right)_{x} \\
& \quad=0
\end{aligned}
$$

where $\gamma, \varepsilon, \kappa, \nu$, and $\delta$ are real parameters. Equation (1) describes the pressure waves in the liquid with gas bubbles taking into account the heat transfer and viscosity [1]. It was called the Kudryashov-Sinelshchikov equation [22].

In practice, analysis of propagation of the pressure waves in a liquid with gas bubbles is an important problem. We know that there are solitary and periodic waves in a mixture of a liquid and gas bubbles, and these waves can be described by the Burgers equation, the Korteweg-de Vries $(\mathrm{KdV})$ equation, and the Burgers-Korteweg-de Vries (BKdV) equation [23-26]. The Kudryashov-Sinelshchikov equation is a generalization of the $\mathrm{KdV}$ and the $\mathrm{BKdV}$ equations. Indeed, assuming that $\varepsilon=\kappa=\delta=0$, we have the Burgers-Kortewegde-Vries equation. In the case of $\varepsilon=\kappa=\lambda=\delta=0$, we get the famous Korteweg-de Vries equation.

Recently, the Kudryashov-Sinelshchikov equation has been investigated by different methods and some exact solutions are derived. Ryabov [22] obtained some exact solutions for $\beta=-3$ and $\beta=-4$ using a modification of the truncated expansion method [27, 28]. Using the bifurcation theory and the method of phase portraits analysis 
$[29,30]$, He et al. [31] investigated bifurcations of travelling wave solutions of the Kudryashov-Sinelshchikov equation and proved the existence of the peakon, solitary wave, and smooth and nonsmooth periodic waves. In this paper, we will derive more new exact Jacobian elliptic function solutions of the Kudryashov-Sinelshchikov equation based on the $F$ expansion method and its extended version.

\section{The F-Expansion Method and Its Extended Version}

In this section, we will give the detailed description of the $F$ expansion method and its extended version.

Suppose that we have a nonlinear partial differential equation (PDE) for $u(x, t)$ in the form

$$
N\left(u, u_{t}, u_{x}, u_{t t}, u_{x t}, u_{x x}, \ldots\right)=0,
$$

where $N$ is a polynomial in its arguments.

By taking $u(x, t)=u(\xi), \xi=x-c t$, we look for traveling wave solutions of (2) and transform it to the ordinary differential equation (ODE)

$$
N\left(u,-c u^{\prime}, u^{\prime}, c^{2} u^{\prime \prime},-c u^{\prime \prime}, u^{\prime \prime}, \ldots\right)=0 \text {. }
$$

Suppose that the solution $u$ of (3) can be expressed as a finite series in the form

$$
u=a_{0}+\sum_{i=1}^{n}\left(a_{i} F^{i}(\xi)+\frac{b_{i}}{F^{i}(\xi)}\right),
$$

where $a_{0}$, and $a_{i}, b_{i}(i=1,2, \ldots, n)$ are constants to be determined later, and $F(\xi)$ is a solution of the auxiliary LODE

$$
F^{\prime 2}(\xi)=P F^{4}(\xi)+Q F^{2}(\xi)+R
$$

where $P, Q$, and $R$ are constants. If the values of $P, Q$, and $R$ are known, the Jacobian elliptic function solutions $F(\xi)$ can be obtained from (5) which can also be found in Table 1.

We may also seek the exact solutions of ODE (3) in the following form:

$$
u=a_{0}+\sum_{i=1}^{n}\left(a_{i} F^{i}(\xi)+b_{i} F^{i-1}(\xi) F^{\prime}(\xi)\right),
$$

where $a_{0}, a_{i}, b_{i}(i=1,2, \ldots, n)$ are constants to be determined later, $F(\xi)$ is a solution of (5).

In the F-expansion method, substituting (4) with (5) into ODE (3) and collecting coefficients of $F^{j}(\xi)(j=$ $0, \pm 1, \pm 2, \ldots)$, we derive a set of overdetermined algebraic equations of $a_{0}, a_{i}$, and $b_{i}$ for $i=1,2, \ldots, n$ by setting each coefficient to zero. Solving these overdetermined algebraic equations by symbolic computation, we can determine those parameters explicitly.

In the extended version of $F$-expansion method, by substituting (5) with (6) into ODE (3) and collecting the coefficients of $F^{j}(\xi) F^{\prime k}(\xi)(j=0, \pm 1, \pm 2, \ldots, k=0,1)$, we derive a set of overdetermined algebraic equations of $a_{0}, a_{i}$, and $b_{i}$ for $i=1,2, \ldots, n$ by setting each coefficient to zero. By solving
TABLE 1: Relations between the coefficients $(P, Q, R)$ and corresponding $f(\xi)$ in $f^{\prime 2}=P f^{4}+Q f^{2}+R[32]$.

\begin{tabular}{lcccc}
\hline Case & $P$ & $Q$ & $R$ & $f(\xi)$ \\
\hline 1 & $m^{2}$ & $-\left(1+m^{2}\right)$ & 1 & $\mathrm{sn} \xi$ \\
2 & $m^{2}$ & $-\left(1+m^{2}\right)$ & 1 & $\mathrm{~cd} \xi$ \\
3 & $-m^{2}$ & $2 m^{2}-1$ & $1-m^{2}$ & $\mathrm{cn} \xi$ \\
4 & -1 & $2-m^{2}$ & $m^{2}-1$ & $\mathrm{dn} \xi$ \\
5 & 1 & $-\left(1+m^{2}\right)$ & $m^{2}$ & $\mathrm{~ns} \xi$ \\
6 & 1 & $-\left(1+m^{2}\right)$ & $m^{2}$ & $\mathrm{dc} \xi$ \\
7 & $1-m^{2}$ & $2 m^{2}-1$ & $-m^{2}$ & $\mathrm{nc} \xi$ \\
8 & $m^{2}-1$ & $2-m^{2}$ & -1 & $\mathrm{nd} \xi$ \\
9 & $1-m^{2}$ & $2-m^{2}$ & 1 & $\mathrm{sc} \xi$ \\
10 & $-m^{2}\left(1-m^{2}\right)$ & $2 m^{2}-1$ & 1 & $\mathrm{sd} \xi$ \\
11 & 1 & $2-m^{2}$ & $1-m^{2}$ & $\mathrm{cs} \xi$ \\
12 & 1 & $2 m^{2}-1$ & $-m^{2}\left(1-m^{2}\right)$ & $\mathrm{ds} \xi$ \\
\hline
\end{tabular}

these overdetermined algebraic equations by symbolic computation, we can determine those parameters explicitly.

Assuming that the constants $a_{0}, a_{i}$, and $b_{i}(i=1,2, \ldots, n)$ can be obtained by solving the algebraic equations, then by substituting these constants and the known general solutions into (4) or (6), we can obtain the explicit solutions of (2) immediately.

\section{Exact Solutions of the Kudryashov- Sinelshchikov Equation in the Case of} $\nu=\delta=0$

In this section, we solve the Kudryashov-Sinelshchikov equation in case of $\nu=\delta=0$ by $F$-expansion method the in order to find the exact solutions of the Kudryashov-Sinelshchikov equation. Using scale transformation:

$$
x=x^{\prime}, \quad t=t^{\prime}, \quad u=\frac{1}{\varepsilon} U,
$$

the Kudryashov-Sinelshchikov equation is written in the form

$$
U_{t}+\alpha U U_{x}+U_{x x x}-\left(U U_{x x}\right)_{x}-\beta U_{x} U_{x x}=0,
$$

where $\alpha=\gamma / \varepsilon$ and $\beta=\kappa / \varepsilon$.

We let

$$
U(x, t)=1-\phi(\xi), \quad \xi=x-c t .
$$

Under this transformation, (8) can be reduced to the following ordinary differential equation (ODE):

$$
c \phi^{\prime}-\alpha(1-\phi) \phi^{\prime}-\phi^{\prime \prime \prime}+\left((1-\phi) \phi^{\prime \prime}\right)^{\prime}-\beta \phi^{\prime} \phi^{\prime \prime}=0 .
$$

By integrating (10) once with respect to $\xi$, we have

$$
\frac{1}{2} \alpha \phi^{2}+(c-\alpha) \phi-\phi \phi^{\prime \prime}-\frac{1}{2} \beta\left(\phi^{\prime}\right)^{2}+C=0,
$$

where $C$ is an integration constant. 
Based on the F-expansion method, we take the solution of ODE (11) as follows:

$$
\phi=a_{0}+a_{1} F(\xi)+\frac{b_{1}}{F(\xi)}+a_{2} F^{2}(\xi)+\frac{b_{2}}{F^{2}(\xi)},
$$

where $a_{0}, a_{1}, b_{1}, a_{2}$, and $b_{2}$ are constants to be determined and $F(\xi)$ satisfies the elliptic Equation (5).

By substituting (12) and (5) into (11), the left-hand side of (11) becomes a polynomial in $F(\xi)$. Setting their coefficients to zero yields a system of algebraic equations in $a_{0}, a_{1}, b_{1}, a_{2}$, and $b_{2}$. By solving the overdetermined algebraic equations by Maple, we can obtain the following six sets of solutions:

(1)

$$
\begin{gathered}
a_{1}=a_{2}=b_{1}=0, \\
c=\frac{-4\left(b_{2}^{2} P+b_{2} Q\left(1-2 a_{0}\right)+3 R a_{0}\left(a_{0}-1\right)\right)}{b_{2}}, \\
\alpha=\frac{4\left(3 R a_{0}-b_{2} Q\right)}{b_{2}}, \\
C=\frac{6 a_{0}\left(b_{2}^{2} P+R a_{0}^{2}-a_{0} b_{2} Q\right)}{b_{2}}, \quad \beta=-3,
\end{gathered}
$$

(2)

$$
\begin{gathered}
a_{1}=b_{1}=b_{2}=0, \\
c=\frac{-4\left(a_{2}^{2} R+a_{2} Q\left(1-2 a_{0}\right)+3 P a_{0}\left(a_{0}-1\right)\right)}{a_{2}}, \\
\alpha=\frac{4\left(3 P a_{0}-a_{2} Q\right)}{a_{2}}, \\
C=\frac{6 a_{0}\left(a_{2}^{2} R+a_{0}^{2} P-a_{0} a_{2} Q\right)}{a_{2}}, \quad \beta=-3,
\end{gathered}
$$

(3)

$$
\begin{gathered}
a_{1}=b_{1}=0, \quad b_{2}=\frac{a_{2} R}{P}, \\
c=\frac{-4\left(-4 a_{2}^{2} R+a_{2} Q\left(1-2 a_{0}\right)+3 P a_{0}\left(a_{0}-1\right)\right)}{a_{2}}, \\
\alpha=\frac{4\left(3 P a_{0}-a_{2} Q\right)}{a_{2}}, \\
C=\frac{6\left(-4 a_{0} a_{2}^{2} R P+4 a_{2}^{3} R Q+a_{0}^{3} P^{2}-a_{0}^{2} P a_{2} Q\right)}{a_{2} P}, \quad \beta=-3,
\end{gathered}
$$

(4)

$$
\begin{gathered}
a_{0}=a_{1}=a_{2}=b_{2}=0, \quad c=-2 Q, \\
\alpha=-2 Q, \quad C=-2 b_{1}^{2} P, \quad \beta=-4,
\end{gathered}
$$

(5)

$$
\begin{gathered}
a_{0}=a_{2}=b_{1}=b_{2}=0, \quad c=-2 Q, \\
\alpha=-2 Q, \quad C=-2 a_{1}^{2} R, \quad \beta=-4,
\end{gathered}
$$

(6)

$$
\begin{gathered}
a_{0}=a_{2}=b_{2}=0, \quad c=-2 Q+12 R \sqrt{\frac{P}{R}}, \\
\alpha=-2 Q+12 R \sqrt{\frac{P}{R}}, \\
C=8 \sqrt{\frac{P}{R}} b_{1}^{2} Q-16 P b_{1}^{2}, \quad \beta=-4 .
\end{gathered}
$$

Substituting (13)-(18) into (12) with (9), we have the following formal solution of (8):

$$
U=1-a_{0}-\frac{b_{2}}{F^{2}(\xi)},
$$

where $\xi=x+\left(4\left(b_{2}^{2} P+b_{2} Q\left(1-2 a_{0}\right)+3 R a_{0}\left(a_{0}-1\right)\right) / b_{2}\right) t$, $\alpha=4\left(3 R a_{0}-b_{2} Q\right) / b_{2}, \beta=-3$,

$$
U=1-a_{0}-a_{2} F^{2}(\xi)
$$

where $\xi=x+\left(4\left(a_{2}^{2} R+a_{2} Q\left(1-2 a_{0}\right)+3 P a_{0}\left(a_{0}-1\right)\right) / a_{2}\right) t$, $\alpha=4\left(3 P a_{0}-a_{2} Q\right) / a_{2}, \beta=-3$,

$$
U=1-a_{0}-a_{2} F^{2}(\xi)-\frac{a_{2} R}{P F^{2}(\xi)},
$$

where $\xi=x+\left(4\left(-4 a_{2}^{2} R+a_{2} Q\left(1-2 a_{0}\right)+3 P a_{0}\left(a_{0}-1\right)\right) / a_{2}\right) t$, $\alpha=4\left(3 P a_{0}-a_{2} Q\right) / a_{2}, \beta=-3$.

$$
U=1-\frac{b_{1}}{F(\xi)},
$$

where $\xi=x+2 Q t, \alpha=-2 Q, \beta=-4$,

$$
U=1-a_{1} F(\xi),
$$

where $\xi=x+2 Q t, \alpha=-2 Q, \beta=-4$, and

$$
U=1-\sqrt{\frac{P}{R}} b_{1} F(\xi)-\frac{b_{1}}{F(\xi)},
$$

where $\xi=x+(2 Q-12 R \sqrt{P / R}) t, \alpha=-2 Q+12 R \sqrt{P / R}$, $\beta=-4$.

Combining (19)-(24) with Table 1, some exact solutions of (8) are obtained.

When $P=m^{2}, Q=-\left(1+m^{2}\right)$, and $R=1$, the solutions of elliptic equation $(5)$ are $F(\xi)=\operatorname{sn}(\xi, m)$ and $F(\xi)=\operatorname{cd}(\xi, m)$ from Table 1 , so the exact solutions for the Kudryashov-Sinelshchikov equation are obtained.

From (19), we have

$$
\begin{aligned}
& U_{1}=1-a_{0}-b_{2} \mathrm{~ns}^{2}(\xi, m), \\
& U_{2}=1-a_{0}-b_{2} \operatorname{dc}^{2}(\xi, m),
\end{aligned}
$$


where $\xi=x+\left(4\left(b_{2}^{2} m^{2}-b_{2}\left(1+m^{2}\right)\left(1-2 a_{0}\right)+3 a_{0}\left(a_{0}-1\right)\right) / b_{2}\right) t$, $\alpha=4\left(3 a_{0}+b_{2}\left(1+m^{2}\right)\right) / b_{2}, \beta=-3$.

When $m \rightarrow 1$, from (25), the exact solution of (8) is

$$
U_{3}=1-a_{0}-b_{2} \operatorname{coth}^{2}(\xi)
$$

where $\xi=x+\left(4\left(b_{2}^{2}-2 b_{2}\left(1-2 a_{0}\right)+3 a_{0}\left(a_{0}-1\right)\right) / b_{2}\right) t, \alpha=$ $4\left(3 a_{0}+2 b_{2}\right) / b_{2}, \beta=-3$.

When $m \rightarrow 0$, from (25) and (26), the exact solutions of (8) are

$$
\begin{aligned}
& U_{4}=1-a_{0}-b_{2} \csc ^{2}(\xi), \\
& U_{5}=1-a_{0}-b_{2} \sec ^{2}(\xi),
\end{aligned}
$$

where $\xi=x+\left(4\left(-b_{2}\left(1-2 a_{0}\right)+3 a_{0}\left(a_{0}-1\right)\right) / b_{2}\right) t, \alpha=4\left(3 a_{0}+\right.$ $\left.b_{2}\right) / b_{2}, \beta=-3$.

From (20), we have

$$
\begin{aligned}
& U_{6}=1-a_{0}-a_{2} \operatorname{sn}^{2}(\xi, m), \\
& U_{7}=1-a_{0}-a_{2} \operatorname{cd}^{2}(\xi, m),
\end{aligned}
$$

where $\xi=x+\left(4\left(a_{2}^{2}-a_{2}\left(1+m^{2}\right)\left(1-2 a_{0}\right)+3 a_{0} m^{2}\left(a_{0}-1\right)\right) / a_{2}\right) t$, $\alpha=4\left(3 a_{0} m^{2}+a_{2}\left(1+m^{2}\right)\right) / a_{2}, \beta=-3$.

When $m \rightarrow 1$, from (29), the exact solution of (8) is

$$
U_{8}=1-a_{0}-a_{2} \tanh ^{2}(\xi)
$$

where $\xi=x+\left(4\left(a_{2}^{2}-2 a_{2}\left(1-2 a_{0}\right)+3 a_{0}\left(a_{0}-1\right)\right) / a_{2}\right) t, \alpha=$ $4\left(3 a_{0}+2 a_{2}\right) / a_{2}, \beta=-3$.

When $m \rightarrow 0$, from (29) and (30), the exact solutions of (8) are

$$
\begin{gathered}
U_{9}=1-a_{0}-a_{2} \sin ^{2}(\xi), \\
U_{10}=1-a_{0}-a_{2} \cos ^{2}(\xi),
\end{gathered}
$$

where $\xi=x+\left(4\left(-a_{2}+2 a_{0} a_{2}+a_{2}^{2}\right) / a_{2}\right) t, \alpha=4, \beta=-3$.

From (21), we have

$$
\begin{aligned}
& U_{11}=1-a_{0}-a_{2} \operatorname{sn}^{2}(\xi, m)-\frac{a_{2}}{m^{2}} \mathrm{~ns}^{2}(\xi, m), \\
& U_{12}=1-a_{0}-a_{2} \mathrm{~cd}^{2}(\xi, m)-\frac{a_{2}}{m^{2}} \mathrm{dc}^{2}(\xi, m),
\end{aligned}
$$

where $\xi=x+\left(4\left(-4 a_{2}^{2}-a_{2}\left(1+m^{2}\right)\left(1-2 a_{0}\right)+3 a_{0} m^{2}\left(a_{0}-\right.\right.\right.$ $\left.1)) / a_{2}\right) t, \alpha=4\left(3 a_{0} m^{2}+a_{2}\left(1+m^{2}\right)\right) / a_{2}, \beta=-3$.

When $m \rightarrow 1$, from (33) the exact solution of (8) is

$$
U_{13}=1-a_{0}-a_{2} \tanh ^{2}(\xi)-a_{2} \operatorname{coth}^{2}(\xi),
$$

where $\xi=x+\left(4\left(-4 a_{2}^{2}-2 a_{2}\left(1-2 a_{0}\right)+3 a_{0}\left(a_{0}-1\right)\right) / a_{2}\right) t$, $\alpha=4\left(3 a_{0}+2 a_{2}\right) / a_{2}, \beta=-3$.

From (22), we have

$$
\begin{aligned}
& U_{14}=1-b_{1} \mathrm{~ns}(\xi, m), \\
& U_{15}=1-b_{1} \mathrm{dc}(\xi, m),
\end{aligned}
$$

where $\xi=x-2\left(1+m^{2}\right) t, \alpha=2\left(1+m^{2}\right), \beta=-4$.
When $m \rightarrow 1$, from (36), the exact solution of $(8)$ is

$$
U_{16}=1-b_{1} \operatorname{coth}(\xi)
$$

where $\xi=x-4 t, \alpha=4, \beta=-4$.

When $m \rightarrow 0$, from (36) and (37), the exact solutions of (8) are

$$
\begin{aligned}
& U_{17}=1-b_{1} \csc (\xi), \\
& U_{18}=1-b_{1} \sec (\xi),
\end{aligned}
$$

where $\xi=x-2 t, \alpha=2, \beta=-4$.

From (23), we have

$$
\begin{aligned}
& U_{19}=1-a_{1} \operatorname{sn}(\xi, m), \\
& U_{20}=1-a_{1} \operatorname{cd}(\xi, m),
\end{aligned}
$$

where $\xi=x-2\left(1+m^{2}\right) t, \alpha=2\left(1+m^{2}\right), \beta=-4$.

When $m \rightarrow 1$, from (40) the exact solution of (8) is

$$
U_{21}=1-a_{1} \tanh (\xi)
$$

where $\xi=x-4 t, \alpha=4, \beta=-4$.

When $m \rightarrow 0$, from (40) and (41), the exact solutions of (8) are

$$
\begin{aligned}
& U_{22}=1-a_{1} \sin (\xi), \\
& U_{23}=1-a_{1} \cos (\xi),
\end{aligned}
$$

where $\xi=x-2 t, \alpha=2, \beta=-4$.

From (24), we have

$$
\begin{aligned}
& U_{24}=1-m b_{1} \operatorname{sn}(\xi, m)-b_{1} \mathrm{~ns}(\xi, m), \\
& U_{25}=1-m b_{1} \mathrm{~cd}(\xi, m)-b_{1} \mathrm{dc}(\xi, m),
\end{aligned}
$$

where $\xi=x-2\left(1+m^{2}+6 m\right) t, \alpha=2\left(1+m^{2}+6 m\right), \beta=-4$.

When $m \rightarrow 1$, from (44) the exact solution of (8) is

$$
U_{26}=1-b_{1} \tanh (\xi)-b_{1} \operatorname{coth}(\xi)
$$

where $\xi=x-16 t, \alpha=16, \beta=-4$.

\section{Exact Solutions of the Kudryashov- Sinelshchikov Equation in the Case of $v \neq 0$, $\delta \neq 0$}

In this section, we solve the Kudryashov-Sinelshchikov equation in case of $v \neq 0, \delta \neq 0$ by the extended version of $F$ expansion method. Using transformation (7), we can write the Kudryashov-Sinelshchikov equation in the following form:

$$
\begin{aligned}
& U_{t}+\alpha U U_{x}+U_{x x x}-\left(U U_{x x}\right)_{x}-\beta U_{x} U_{x x}-\nu U_{x x}-\mu\left(U U_{x}\right)_{x} \\
& =0
\end{aligned}
$$

where $\alpha=\gamma / \varepsilon, \beta=\kappa / \varepsilon, \mu=\delta / \varepsilon$. 
We let

$$
U(x, t)=1-\phi(\xi), \quad \xi=x-c t .
$$

Under this transformation, (47) can be reduced to the following ordinary differential equation (ODE):

$$
\begin{aligned}
& c \phi^{\prime}-\alpha(1-\phi) \phi^{\prime}-\phi^{\prime \prime \prime}+\left((1-\phi) \phi^{\prime \prime}\right)^{\prime} \\
& -\beta \phi^{\prime} \phi^{\prime \prime}+\nu \phi^{\prime \prime}-\mu\left((\phi-1) \phi^{\prime}\right)^{\prime}=0 .
\end{aligned}
$$

By integrating (49) once with respect to $\xi$, we have

$$
\begin{aligned}
\frac{1}{2} \alpha \phi^{2} & +(c-\alpha) \phi-\phi \phi^{\prime \prime}-\frac{1}{2} \beta\left(\phi^{\prime}\right)^{2}+\nu \phi^{\prime}-\mu(\phi-1) \phi^{\prime}+C \\
& =0
\end{aligned}
$$

where $C$ is integration constant.

Based on the extended version of $F$-expansion method, we take the solution of ODE (50) as follows:

$$
\phi=a_{0}+a_{1} F(\xi)+a_{2} F^{2}(\xi)+b_{1} F^{\prime}(\xi)+b_{2} F(\xi) F^{\prime}(\xi),
$$

where $a_{0}, a_{1}, b_{1}, a_{2}$, and $b_{2}$ are constants to be determined, and $F(\xi)$ satisfies the elliptic Equation (5).

Substituting (51) and (5) into (50), the left-hand side of (50) becomes a polynomial in $F^{j}(\xi) F^{\prime k}(\xi)(j=0, \pm 1, \pm 2, \ldots$, $k=0,1)$. Setting their coefficients to zero yields a system of algebraic equations in $a_{0}, a_{1}, b_{1}, a_{2}$, and $b_{2}$ By solving the overdetermined algebraic equations by Maple, we can obtain the following solution:

$$
\begin{gathered}
a_{0}=a_{1}=b_{1}=0, \quad a_{2}=\sqrt{\mathrm{Q}} b_{2}, \\
c=-\frac{4}{3}\left(5 \sqrt{\mathrm{Q}} R b_{2}+4 Q\right), \quad \mu=-\frac{2}{3} \sqrt{\mathrm{Q}}, \\
\nu=\frac{2}{3}\left(\sqrt{\mathrm{Q}}+2 R b_{2}\right), \quad \alpha=-\frac{16 Q}{3}, \\
\beta=-\frac{8}{3}, \quad C=-\frac{8}{3} R^{2} b_{2}^{2} .
\end{gathered}
$$

Substituting (52) into (51) with (48), we have the following formal solution of (47):

$$
U=1-\sqrt{\mathrm{Q}} b_{2} F^{2}(\xi)-b_{2} F(\xi) F^{\prime}(\xi),
$$

where $\xi=x+(4 / 3)\left(5 \sqrt{Q} R b_{2}+4 Q\right) t, \mu=-(2 / 3) \sqrt{Q}, v=$ $(2 / 3)\left(\sqrt{Q}+2 R b_{2}\right), \alpha=-16 Q / 3, \beta=-8 / 3$.

By combining (53) with Table 1, some exact solutions of (47) are obtained.
When $P=m^{2}, Q=-\left(1+m^{2}\right), R=1$, the solutions of elliptic equation $(5)$ are $F(\xi)=\operatorname{sn}(\xi, m)$ and $F(\xi)=\operatorname{cd}(\xi, m)$ from Table 1, so the exact solutions of (47) are

$$
\begin{aligned}
U_{1}=1-b_{2}( & i \sqrt{1+m^{2}} \operatorname{sn}^{2}(\xi, m) \\
& +\operatorname{sn}(\xi, m) \mathrm{cn}(\xi, m) \mathrm{dn}(\xi, m)), \\
U_{2}=1-b_{2}( & i \sqrt{1+m^{2}} \operatorname{cd}^{2}(\xi, m) \\
& \left.-\left(1-m^{2}\right) \mathrm{cd}(\xi, m) \operatorname{sd}(\xi, m) \operatorname{nd}(\xi, m)\right),
\end{aligned}
$$

where $\xi=x-(4 / 3)\left(4\left(1+m^{2}\right)-5 b_{2} i \sqrt{1+m^{2}}\right) t, \mu=$ $-(2 / 3) i \sqrt{1+m^{2}}, v=(2 / 3)\left(i \sqrt{1+m^{2}}+2 b_{2}\right), \alpha=(16 / 3)(1+$ $\left.m^{2}\right), \beta=-8 / 3$.

When $m \rightarrow 1$, from (54), the exact solution of (47) is

$$
U_{3}=1-b_{2}\left(i \sqrt{2} \tanh ^{2}(\xi)+\tanh (\xi) \operatorname{sech}^{2}(\xi)\right),
$$

where $\xi=x-(4 / 3)\left(8-5 b_{2} i \sqrt{2}\right) t, \mu=-(2 / 3) i \sqrt{2}, v=$ $(2 / 3)\left(i \sqrt{2}+2 b_{2}\right), \alpha=32 / 3, \beta=-8 / 3$.

When $m \rightarrow 0$, from (54) and (55), the exact solutions of (47) are

$$
\begin{aligned}
& U_{4}=1-b_{2}\left(i \sin ^{2}(\xi)+\sin (\xi) \cos (\xi)\right), \\
& U_{5}=1-b_{2}\left(i \cos ^{2}(\xi)-\cos (\xi) \sin (\xi)\right),
\end{aligned}
$$

where $\xi=x-(4 / 3)\left(4-5 b_{2} i\right) t, \mu=-(2 / 3) i, v=(2 / 3)(i+$ $\left.2 b_{2}\right) \quad \alpha=16 / 3, \beta=-8 / 3$.

When $P=-m^{2}, Q=2 m^{2}-1$, and $R=1-m^{2}$, the solutions of elliptic Equation (5) are $F(\xi)=\mathrm{cn}(\xi, m)$ from Table 1, so the exact solution of (47) is

$$
\begin{aligned}
U_{6}=1-b_{2}( & \sqrt{2 m^{2}-1} \mathrm{cn}^{2}(\xi, m) \\
& -\operatorname{cn}(\xi, m) \operatorname{dn}(\xi, m) \operatorname{sn}(\xi, m)),
\end{aligned}
$$

where $\xi=x+(4 / 3)\left(5 b_{2} \sqrt{2 m^{2}-1}\left(1-m^{2}\right)+4\left(2 m^{2}-1\right)\right) t$, $\mu=-(2 / 3) \sqrt{2 m^{2}-1}, \quad \nu=(2 / 3)\left(\sqrt{2 m^{2}-1}+2 b_{2}\left(1-m^{2}\right)\right)$, $\alpha=(16 / 3)\left(1-2 m^{2}\right), \beta=-8 / 3$.

When $m \rightarrow 1$, from (58) the exact solution of (47) is

$$
U_{7}=1-b_{2}\left(\operatorname{sech}^{2}(\xi, m)-\operatorname{sech}^{2}(\xi) \tanh (\xi)\right),
$$

where $\xi=x+(16 / 3) t, \mu=-2 / 3, v=2 / 3, \alpha=-16 / 3, \beta=$ $-8 / 3$.

When $P=1-m^{2}, Q=2-m^{2}$, and $R=1$, the solutions of elliptic Equation (5) are $F(\xi)=s c(\xi, m)$ from Table 1, so the exact solution of (47) is

$$
\begin{aligned}
U_{8}=1-b_{2}( & \sqrt{2-m^{2}} \operatorname{sc}^{2}(\xi, m) \\
& +\mathrm{dc}(\xi, m) \operatorname{sc}(\xi, m) \mathrm{nc}(\xi, m)),
\end{aligned}
$$


where $\xi=x+(4 / 3)\left(5 b_{2} \sqrt{2-m^{2}}+4\left(2-m^{2}\right)\right) t, \mu=$ $-(2 / 3) \sqrt{2-m^{2}}, v=(2 / 3)\left(\sqrt{2-m^{2}}+2 b_{2}\right), \alpha=(16 / 3)\left(m^{2}-\right.$ 2), $\beta=-8 / 3$.

When $m \rightarrow 1$, from (60), the exact solution of (47) is

$$
U_{9}=1-b_{2}\left(\sinh ^{2}(\xi)+\sinh (\xi) \cosh (\xi)\right),
$$

where $\xi=x+(4 / 3)\left(5 b_{2}+4\right) t, \mu=-(2 / 3), v=(2 / 3)\left(1+2 b_{2}\right)$, $\alpha=-16 / 3, \beta=-8 / 3$.

When $m \rightarrow 0$, from (60), the exact solution of (47) is

$$
U_{10}=1-b_{2}\left(\sqrt{2} \tan ^{2}(\xi)+\sec ^{2}(\xi) \tan (\xi)\right),
$$

where $\xi=x+(4 / 3)\left(5 \sqrt{2} b_{2}+8\right) t, \mu=-(2 / 3) \sqrt{2}, \nu=(2 / 3)$ $\left(\sqrt{2}+2 b_{2}\right), \alpha=-32 / 3, \beta=-8 / 3$.

\section{Conclusions}

The F-expansion method and its extended version are very effective in solving various NLEEs. For some NLEEs, the F-expansion method can give nontrivial solutions, for some other NLEEs, the extended version of F-expansion method can give nontrivial solutions, and for some particular NLEEs (especially the complete integrable systems), both Fexpansion method and its extended version are feasible for constructing exact solutions.

In summary, lots of new exact Jacobian elliptic function solutions and soliton solutions of the KudryashovSinelshchikov equation are proposed by the F-expansion method and its extended version. The results of [21, 22] have been enriched. These exact solutions have been verified by symbolic computation system-Maple. Moreover, the solutions listed in this paper may be of important significance for the explanation of some relevant physical problems. We would like to study the Kudryashov-Sinelshchikov equation further.

\section{Acknowledgments}

This work was supported by the National Natural Science Foundation of China (11161020), the Natural Science Foundation of Yunnan Province (2011FZ193), and the Natural Science Foundation of Education Committee of Yunnan Province (2012Y452).

\section{References}

[1] M. L. Wang, Y. B. Zhou, and Z. B. Li, "Application of a homogeneous balance method to exact solutions of nonlinear equations in mathematical physics," Physics Letters, vol. 216, no. 1-5, pp. 67-75, 1996.

[2] M. L. Wang, "Exact solutions for a compound KdV-Burgers equation," Physics Letters A, vol. 213, no. 5-6, pp. 279-287, 1996.

[3] S. Zhang and T. C. Xia, "A generalized new auxiliary equation method and its applications to nonlinear partial differential equations," Physics Letters A, vol. 363, no. 5-6, pp. 356-360, 2007.

[4] Sirendaoreji and S. Jiong, "Auxiliary equation method for solving nonlinear partial differential equations," Physics Letters A, vol. 309, no. 5-6, pp. 387-396, 2003.
[5] X.-H. (Benn) Wu and J.-H. He, "EXP-function method and its application to nonlinear equations," Chaos, Solitons \& Fractals, vol. 38, no. 3, pp. 903-910, 2008.

[6] X.-H. (Benn) Wu and J.-H. He, "Solitary solutions, periodic solutions and compacton-like solutions using the Exp-function method," Computers \& Mathematics with Applications, vol. 54, no. 7-8, pp. 966-986, 2007.

[7] H.-C. Hu, X.-Y. Tang, S.-Y. Lou, and Q.-P. Liu, "Variable separation solutions obtained from Darboux transformations for the asymmetric Nizhnik-Novikov-Veselov system," Chaos, Solitons \& Fractals, vol. 22, no. 2, pp. 327-334, 2004.

[8] S. B. Leble and N. V. Ustinov, "Darboux transforms, deep reductions and solitons," Journal of Physics A, vol. 26, no. 19, pp. 5007-5016, 1993.

[9] H. A. Abdusalam, "On an improved complex tanh-function method," International Journal of Nonlinear Sciences and Numerical Simulation, vol. 6, no. 2, pp. 99-106, 2005.

[10] J. Lee and R. Sakthivel, "New exact travelling wave solutions of bidirectional wave equations," Pramana Journal of Physics, vol. 76, no. 6, pp. 819-829, 2011.

[11] E. J. Parkes, B. R. Duffy, and P. C. Abbott, "The Jacobi elliptic-function method for finding periodic-wave solutions to nonlinear evolution equations," Physics Letters A, vol. 295, no. 5-6, pp. 280-286, 2002.

[12] S. K. Liu, Z. T. Fu, S. D. Liu, and Q. Zhao, "Jacobi elliptic function expansion method and periodic wave solutions of nonlinear wave equations," Physics Letters A, vol. 289, no. 1-2, pp. 69-74, 2001.

[13] Y. B. Zhou, M. L. Wang, and Y. M. Wang, "Periodic wave solutions to a coupled KdV equations with variable coefficients," Physics Letters A, vol. 308, no. 1, pp. 31-36, 2003.

[14] M. L. Wang and X. Z. Li, "Applications of F-expansion to periodic wave solutions for a new Hamiltonian amplitude equation," Chaos, Solitons and Fractals, vol. 24, no. 5, pp. 1257$1268,2005$.

[15] W.-W. Li, Y. Tian, and Z. Zhang, "F-expansion method and its application for finding new exact solutions to the sineGordon and sinh-Gordon equations," Applied Mathematics and Computation, vol. 219, no. 3, pp. 1135-1143, 2012.

[16] S. Zhang and T. C. Xia, "A generalized F-expansion method and new exact solutions of Konopelchenko-Dubrovsky equations," Applied Mathematics and Computation, vol. 183, no. 2, pp. 11901200,2006

[17] Y.-J. Ren and H.-Q. Zhang, "A generalized F-expansion method to find abundant families of Jacobi elliptic function solutions of the $(2+1)$-dimensional Nizhnik-Novikov-Veselov equation," Chaos, Solitons and Fractals, vol. 27, no. 4, pp. 959-979, 2006.

[18] G. L. Cai, Q. C. Wang, and J. J. Huang, "A modified F-expansion method for solving breaking soliton equation," International Journal of Nonlinear Science, vol. 2, no. 2, pp. 122-128, 2006.

[19] E. Yomba, "The extended F-expansion method and its application for solving the nonlinear wave, CKGZ, GDS, DS and GZ equations," Physics Letters A, vol. 304, no. 1-4, pp. 149-160, 2005.

[20] J. L. Zhang, M. L. Wang, Y. M. Wang, and Z. D. Fang, "The improved F-expansion method and its applications," Physics Letters A, vol. 350, no. 1-2, pp. 103-109, 2006.

[21] N. A. Kudryashov and D. I. Sinelshchikov, "Nonlinear waves in bubbly liquids with consideration for viscosity and heat transfer," Physics Letters A, vol. 374, no. 19-20, pp. 2011-2016, 2010. 
[22] P. N. Ryabov, "Exact solutions of the Kudryashov-Sinelshchikov equation," Applied Mathematics and Computation, vol. 217, no. 7, pp. 3585-3590, 2010.

[23] D. J. Korteweg and G. de Vries, "On the change of long waves advancing in a rectangular canal, and on a new type of long stationary waves," Philosophical Magazine Series, vol. 39, no. 240, pp. 422-443, 1895.

[24] G. B. Whitham, Linear and Nonlinear Waves, Wiley-Interscience, New York, NY, USA, 1974.

[25] V. E. Nakoryakov, V. V. Sobolev, and L. R. Shreiber, "Long waves perturbations in a gas-liquid mixture," Fluid Dynamics, vol. 7, pp. 763-768, 1972.

[26] N. A. Kudryashov, "Exact solitary waves of the Fisher equation," Physics Letters A, vol. 342, no. 1-2, pp. 99-106, 2005.

[27] N. A. Kudryashov, "One method for finding exact solutions of nonlinear differential equations," Communications in Nonlinear Science and Numerical Simulation, vol. 17, no. 6, pp. 2248-2253, 2012.

[28] P. N. Ryabov, D. I. Sinelshchikov, and M. B. Kochanov, "Application of the Kudryashov method for finding exact solutions of the high order nonlinear evolution equations," Applied Mathematics and Computation, vol. 218, no. 7, pp. 3965-3972, 2011.

[29] J. Li, Y. Zhang, and G. Chen, "Exact solutions and their dynamics of traveling waves in three typical nonlinear wave equations," International Journal of Bifurcation and Chaos in Applied Sciences and Engineering, vol. 19, no. 7, pp. 2249-2266, 2009.

[30] H. Bin, L. Jibin, L. Yao, and R. Weiguo, "Bifurcations of travelling wave solutions for a variant of Camassa-Holm equation," Nonlinear Analysis. Real World Applications, vol. 9, no. 2, pp. 222-232, 2008.

[31] B. He, Q. Meng, and Y. Long, "The bifurcation and exact peakons, solitary and periodic wave solutions for the Kudryashov-Sinelshchikov equation," Communications in Nonlinear Science and Numerical Simulation, vol. 17, no. 11, pp. 4137-4148, 2012.

[32] M. A. Abdou, "The extended F-expansion method and its application for a class of nonlinear evolution equations," Chaos, Solitons \& Fractals, vol. 31, no. 1, pp. 95-104, 2007. 


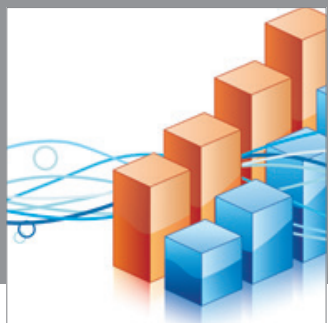

Advances in

Operations Research

mansans



The Scientific World Journal
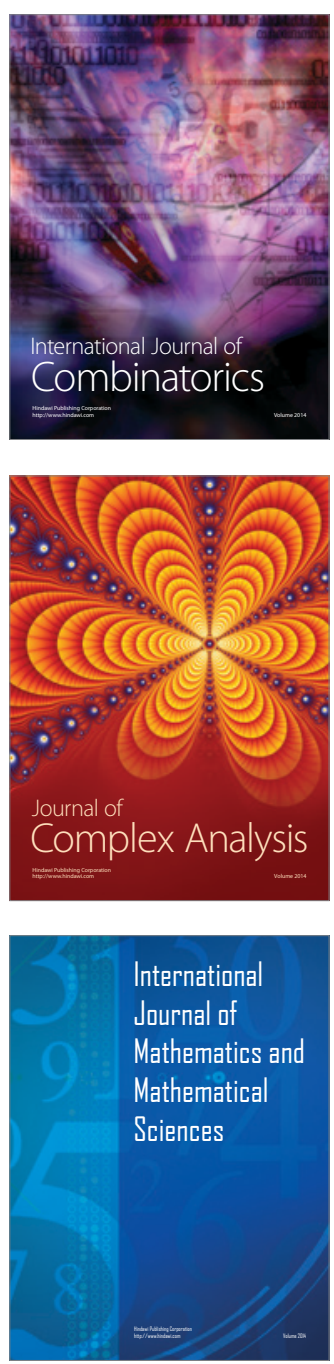
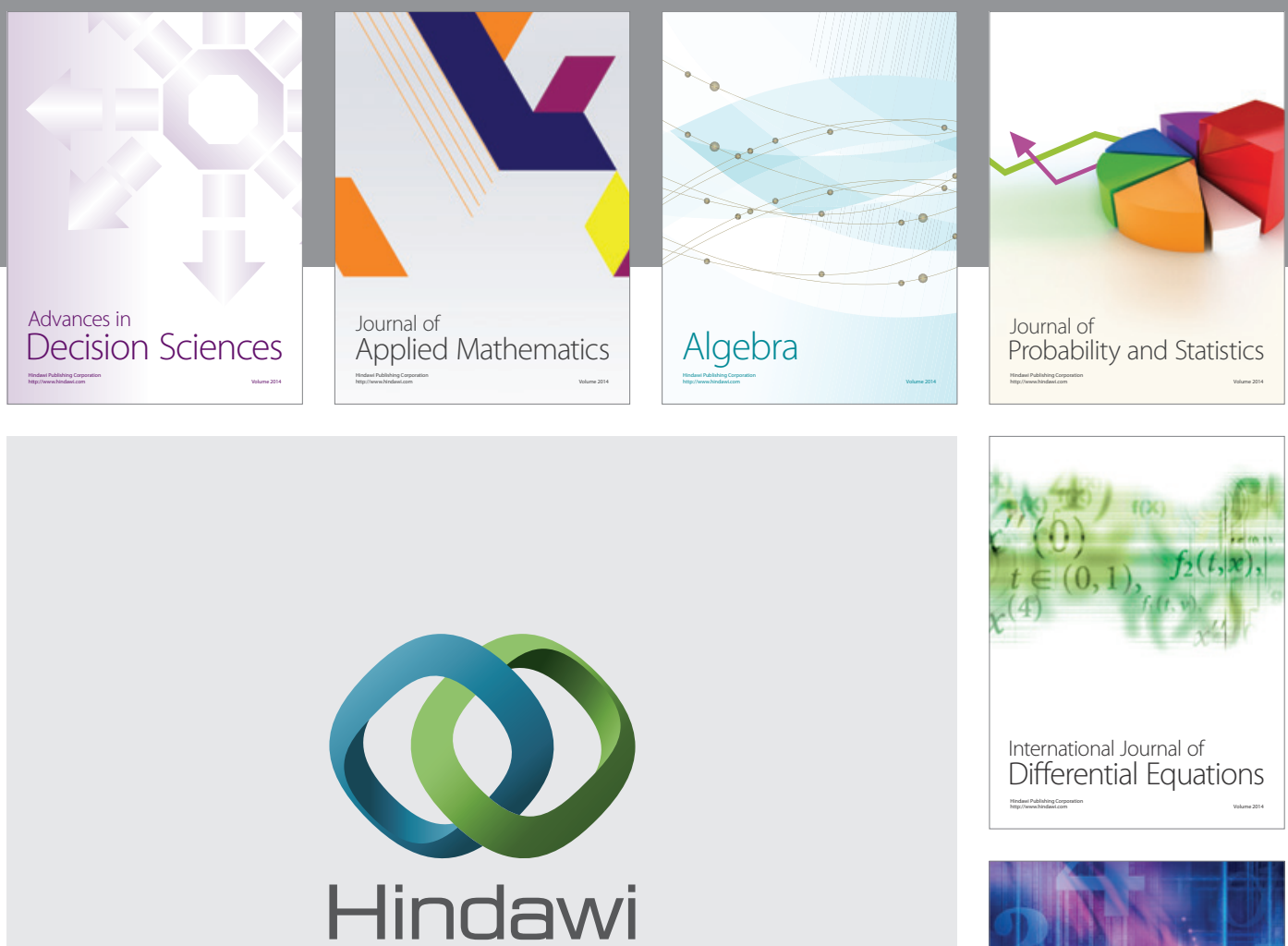

Submit your manuscripts at http://www.hindawi.com
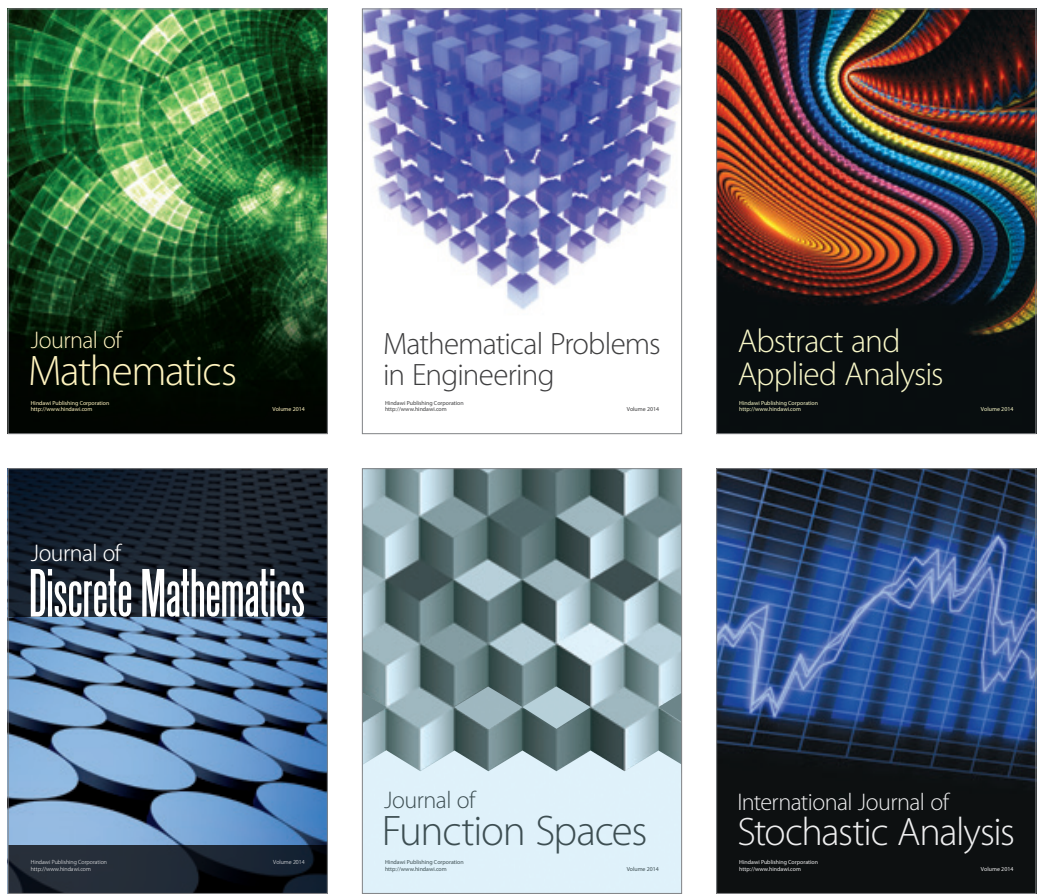

Journal of

Function Spaces

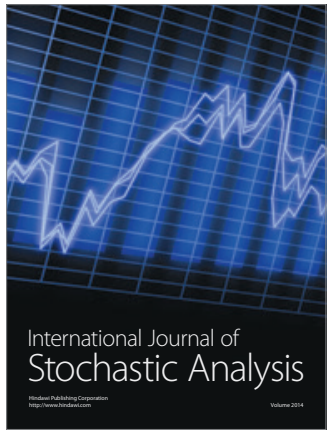

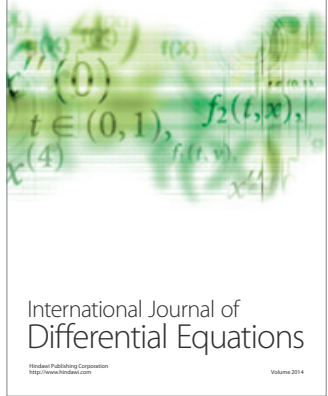
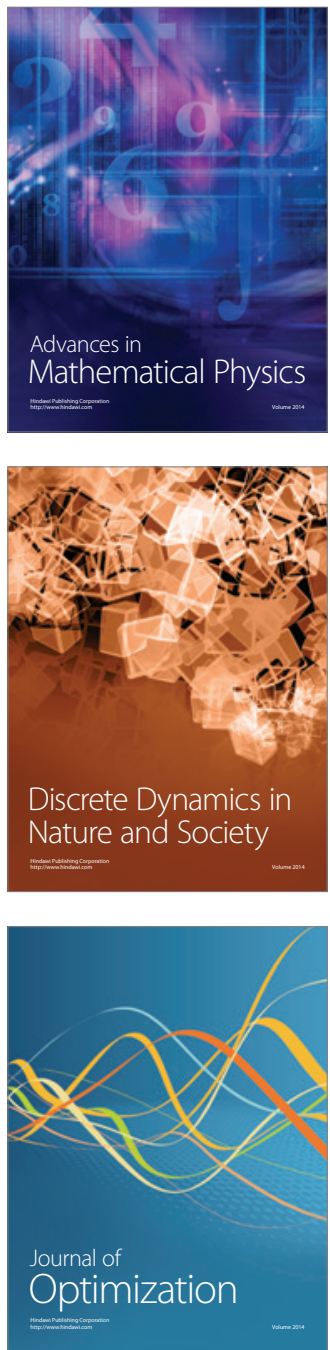